\title{
How to Prepare Manuscripts for Publication in Journal of Materials Research
}

Journal of Materials Research (JMR) is an archival journal of leading-edge interdisciplinary research on materials. It is published by the Materials Research Society and its subscribers include the more than 7,500 MRS members and hundreds of scientific and technical libraries around the world. Prior to publication, each JMR manuscript is thoroughly reviewed under the supervision of one of JMR's Principal Editors, all recognized experts in their respective fields. These reviews guarantee that articles appearing in JMR are important new contributions to materials science.

Joumal of Materials Research strives to provide a rapid turnaround from submittal to publication for each manuscript without sacrificing quality. (This is critical for manuscripts in rapidly growing fields such as high temperature superconductors.) Authors can help ensure a more rapid turnaround for their $J M R$ manuscripts by closely following $J M R$ 's "Information for Contributors." These guidelines, published in $J M R$ 's January/February issue each year, are designed to maintain high standards of quality as well as uniformity among articles published in $J M R$. By adhering to these instructions for manuscript preparation, authors can help ensure prompt publication of accepted papers.

The "Instructions for Contributors" to $J M R$ are reprinted in this issue of the BULLETIN for your convenience. Additional questions concerning manuscript preparation or submittal can be directed to:

Linda A. Krysinski, Editorial Office

Supervisor

Journal of Materials Research

c/o Materials Research Society

9800 McKnight Road, Suite 327

Pittsburgh, PA 15237

Telephone (412) 367-9111

Fax (412) 367-4373

\section{Information for Contributors to Journal of Materials Research}

1. Articles. Joumal of Materials Research publishes original research reports that include complete, detailed, self-contained descriptions of research efforts. All articles must contain an abstract (see item 4).

2. Rapid Communications. Joumal of Materials Research also publishes short submissions (i.e., not more than four published pages), which contain important new results and are intended for acceler- ated publication. Rapid Communications are given priority in handling. An abstract is required.

3. Commentaries and Reviews. Joumal of Materials Research occasionally publishes commentaries on topics of current interest or reviews of the literature in a given area. An abstract is required.

4. Abstract. An abstract must accompany each manuscript. The abstract should be typed (double-spaced) on a separate page. It should be adequate as an index and as a summary. It should give all subjects, major and minor, concerning which new information is presented. It should give the conclusions of the article and all numerical results of general interest. The abstract should avoid the use of jargon and other terminology that would render its content inaccessible to the nonspecialist. The abstract is usually reprinted verbatim in abstract journals.

All abstracts are indexed according to a materials classification scheme and a topical keyword listing. Descriptions of both indexing procedures are available from the JMR Editorial Office. Authors are expected to provide the Editorial Office with suitable index classifications for their manuscripts prior to acceptance of any manuscript for publication.

Authors may also classify their work according to the 1988 version of the Physics and Astronomy Classification Scheme (PACS). PACS renders an author's work more readily available in automated retrieval systems, provided the subject matter of the work is appropriate for the PACS scheme. Authors' identification of PACS classification is optional, and if authors do not indicate a PACS classification, one will be provided for them. A free booklet on the PACS scheme is available from the PACS Editor at the American Institute of Physics. (See AIP address in item 6.)

5. Manuscripts. Manuscripts are accepted with the understanding that they have not been copyrighted, published, or accepted for publication elsewhere. Manuscripts must be in English, typewritten, double-spaced on one side of the page, on $8-1 / 2 \times 11$ in. durable, opaque, white paper. An original and three (3) copies must be submitted. Margins should be a minimum of $1 \mathrm{in}$. on both sides of the paper to permit editorial notations. Authors should use an identical format for their names in all publications to facilitate use of citations and author indexes. Manuscripts must be submitted with a cover letter containing the name, address, and telephone number of the author to whom correspondence should be addressed.

6. Style. Authors are expected to follow the conventional writing, notation, and illustration style prescribed in the American Institute of Physics Style Manual, which is available from the AIP, 335 East 45th St., New York, NY 10017 for $\$ 7.50$ prepaid. Authors should also study the form and style of printed material in any issue of JMR. SI units should be used.

7. Illustrations. All illustrations must accompany submitted manuscripts and, like the text, must be in quadruplicate (one original and three copies). Every illustration must be referred to in the main text in consecutive numerical order. A caption (legend) must accompany each illustration. Captions must be typed doublespaced on a sheet of paper at the end of the manuscript. Drawings should be planned with oversize lettering and wide- spaced coordinates to be reproduced satisfactorily within single-column width $(80 \mathrm{~mm}$, or $3-$ $1 / 8$ in.). Principal letters on figures are reduced to approximately $1.5 \mathrm{~mm}$ or $1 / 16 \mathrm{in}$. or, if a Leroy lettering device is used, to $\# 060$, regardless of the overall size of the drawing. For best results, draftsmen should be given a recent copy of joumal of Materials Research to familiarize themselves with the proportions to be followed.

Line drawings are preferable to photographs and must be made with India ink on plain white paper or tracing cloth. Coordinate paper is not acceptable. Illustrations must be held, with rarest exceptions, to 8$1 / 2 \times 11$ in. High-quality glossy prints with sharp even lines and lettering may be submitted in place of original drawings when these are of exaggerated size and awkward to handle.

Photographs of standard apparatus in a laboratory setup are not instructive and should not be included. No heavy writing should be made on the backs of photographs nor should they be paper clipped or stapled, which may result in damage to the picture. The figure number and the author's name should appear on the back of each illustration.

All photographs, including micro- 
graphs, should be good quality, continuous tone black-and-white prints. Micrographs should contain a fiducial mark that indicates explicitly the scale length that is being shown. Specification of the reduction ratio in the caption is not adequate. Any arrows or lettering in the micrograph must satisfy the same scaling restrictions as those indicated above for line drawings.

Unless necessary, all photographs will be reduced to single-column width. Any special instructions regarding the scaling or placement of photographs should be written on the back of all the photographs involved.

Authors must assume responsibility for the quality of computer-generated graphs or charts. It is necessary to exercise control over the computer-driven printer so that the lines of a graph are dark enough for reproduction. If the labeling on a computer-generated plot is of poor quality, professionally hand-lettered characters should be superimposed on the plot. Computer-generated plots made up of small horizontal and vertical line segments may be rejected if the segments are not short enough.

8. Mathematics. Mathematical expressions should be typewritten as completely as possible, with unavailable symbols being carefully inserted in ink. Special care should be given to make equations and formulas clear to the typesetter, and each letter or symbol inserted in the manuscript in handwriting should be identified in the margin the first time it occurs in the text. Capital and lower-case letters should be distinguished clearly where there could be confusion. Fractional exponents should be used to avoid root signs. Extra symbols should be introduced to avoid complicated exponents or when it is necessary to repeat a complicated expression a number of times. The solidus (/) should be used wherever possible for fractions. Mathematical derivations that are easily found elsewhere in the literature should not be used. Rather the references should be cited.

9. References. References should be collected and typed (double-spaced) on a separate page. They should be numbered consecutively and arranged thus:

'A.B. Smith, Phys. Rev. 41, 852 (1932). 2H. Lamb, Hydrodynamics (Cambridge U.P., Cambridge, England, 1940), 6th ed., pp. 573,645

A list of the abbreviations for the names of journals appears in the American Institute of Physics Style Manual (see item 6).
10. Tables. All but the simplest tabular material should be organized into separate tables. Tables should be numbered with Roman numerals and typed on sheets at the end of the manuscript. Captions should be sufficiently descriptive to make the data in the table intelligible without referring to the text. Complicated column headings in the body of the table should be avoided. If necessary, symbols that are explained in the caption should be used. A double horizontal line should be below the caption, a single line below the headings, and another double line at the end of the table. Long tables should, if possible, be submitted in a form ready for direct photoreproduction. Photoreproducible tables (including captions) should be typed single-spaced on an electric typewriter with Elite type or an equivalent proportional-spaced typeface, and should be designed to fit into one journal column $(80 \times 248 \mathrm{~mm}$, or $3-1 / 8 \times 9-3 / 4 \mathrm{in}$.) or across two columns $(167 \times 248 \mathrm{~mm}$, or $6-5 / 8 \times 9-3 / 4$ in.). Since they will be photoreduced by a factor of 0.75 , photoreduction should be anticipated by making the type-area dimensions of the table $4 / 3$ larger than the final column and page dimensions given above. Detailed instructions for preparing camera-ready tables are available from the JMR Editorial Office.

11. Publication Charges defray a major portion of the cost of the production of this journal. Consequently, authors' institutions or companies are urged to pay a publication charge of $\$ 70$ per printed page, which entitles them to 100 reprints. An appropriate form will be mailed to authors upon acceptance of the manuscript.

12. Proofs of Articles will be sent to the author for review and should be returned promptly as instructed. A few alterations in proof are unavoidable, but the cost of making extensive alterations or of correcting mistakes caused by careless preparation of the manuscript will be charged to the author. Corrected proofs which are not returned punctually may result in a delay in publication of the paper.

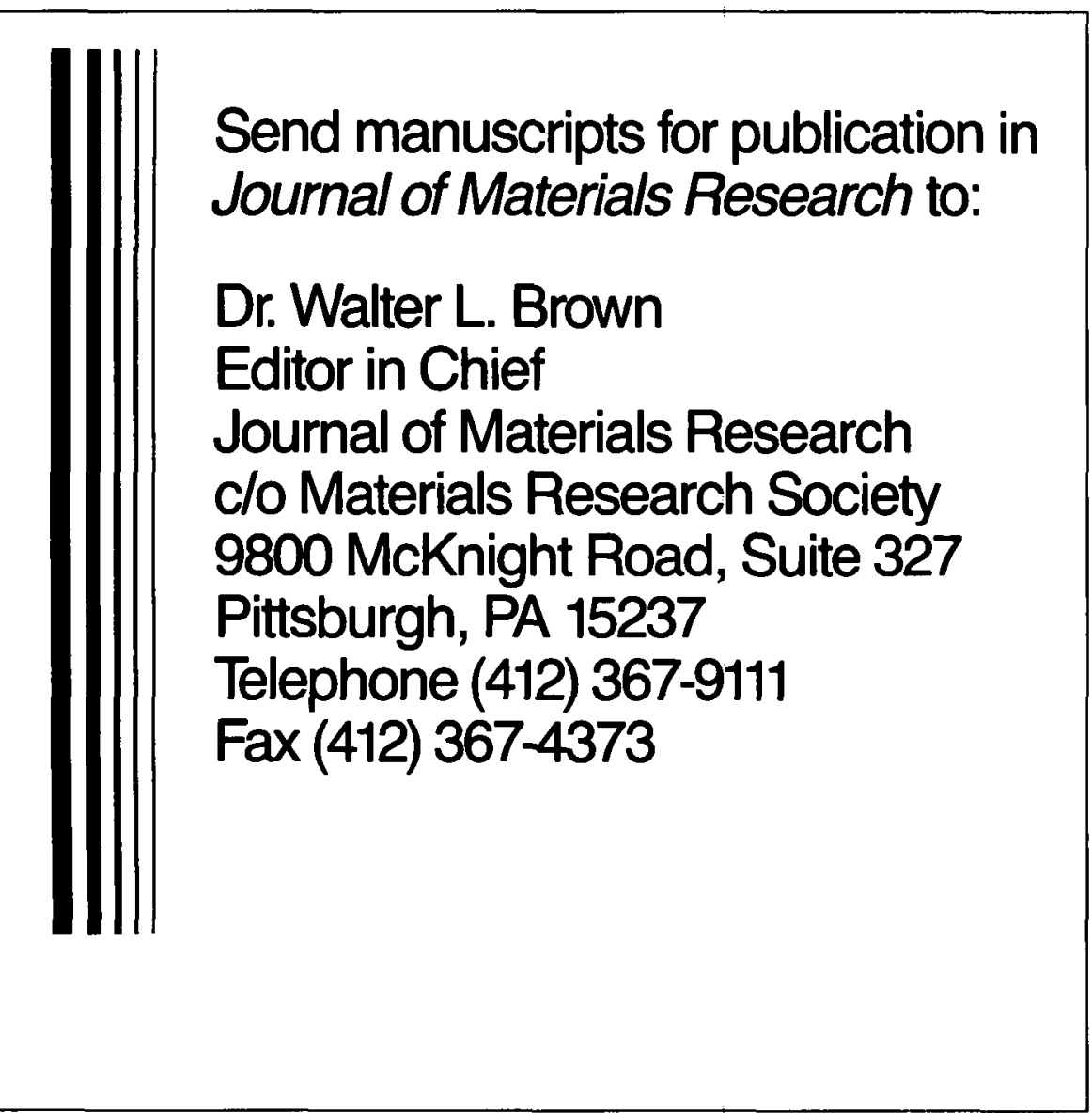

